SIT-HEP/TM-15

\title{
Hybridized Affleck-Dine baryogenesis
}

\author{
Tomohiro Matsuda ${ }^{1}$ \\ Laboratory of Physics, Saitama Institute of Technology, \\ Fusaiji, Okabe-machi, Saitama 369-0293, Japan
}

\begin{abstract}
We propose a novel scenario for Affleck-Dine baryogenesis in the braneworld, considering the hybrid potential for the Affleck-Dine field. Destabilization of the flat direction is not due to the Hubble parameter, but is induced by a trigger field. The moduli for the brane distance plays the role of the trigger field. Q-balls are unstable in models with large extra dimensions.
\end{abstract}

\footnotetext{
${ }^{1}$ matsuda@sit.ac.jp
} 


\section{Introduction}

In spite of the great success in the quantum field theory, there is still no consistent scenario in which quantum gravity is successfully included. Perhaps the most promising scenario in this direction would be the string theory, in which the consistency is ensured by the requirement of the additional dimensions. In the old scenarios where no branes are included, sizes of the extra dimensions had been assumed to be as small as $M_{p}^{-1}$. However, later observations showed that there is no reason to believe such tiny compactification radius [1]. In models with large extra dimensions, the observed Planck mass is obtained by the relation $M_{p}^{2}=M_{*}^{n+2} V_{n}$, where $M_{*}$ and $V_{n}$ denote the fundamental scale of gravity and the volume of the $n$-dimensional compact space. In this respect, what we had seen in the old string theory was a tiny part of the whole story. In the new scenario, the compactification radius (or the fundamental scale) is the unknown parameter that should be determined by observations. Until recently, cosmological models for such large compactification radius had not been discussed. ${ }^{2}$ Constructing models for the particle cosmology with large extra dimensions is very important since future cosmological observations will play important roles in determining the underlying theory.

In this paper we consider novel ways to realize Affleck-Dine baryogenesis [2] in models with the fundamental scale that is much lower than the conventional GUT scale. Of course, there are drastic changes from the conventional scenarios. In the conventional scenarios of Affleck-Dine baryogenesis, it had been found that the dynamics of the Affleck-Dine field is rather complicated because of the non-trivial formation and decay of Q-balls [3]. In generic cases, almost all the produced baryon number is absorbed into Q-balls. Their properties depend on how supersymmetry breaking is transmitted. If supersymmetry breaking is mediated by gravity, Q-balls are semi-stable but long-lived and may be the source of all the baryons and LSP dark matter 8 . For the gauge-mediated scenarios, Q-balls can be stable and form dark matter that can be searched for directly. Unstable Q-balls can decay to provide all the baryonic charges trapped inside Q-balls, if some requirements are fulfilled. If Q-balls are absolutely stable, it is difficult to produce

\footnotetext{
${ }^{2}$ Constructing successful models for inflation with the low fundamental scale is an interesting problem 4, 11. Baryogenesis in models with the low fundamental scale is discussed in ref. [5, 6, 7].
} 
the sufficient baryon in the plasma although huge baryon number will be kept inside Qballs. It is also a problem if Q-balls decay late to produce unwanted relics in the Universe. On the other hand, when one considers models with the low fundamental scale, the gravitino mass $\left(m_{3 / 2}\right)$ is much smaller than the soft breaking mass $\left(m_{\text {soft }}\right)$ in the sector of the minimal supersymmetric standard model (MSSM). In this respect, the situation looks similar to the gauge-mediated supersymmetry breaking (GMSB) scenarios. In GMSB models, however, the flat potential for the Affleck-Dine mechanism becomes very flat at large amplitudes. It is schematically given by the formula

$$
\begin{aligned}
V_{G M S B} & \sim m_{\text {soft }}^{2}\left|\phi_{A D}\right|^{2}\left(\phi_{A D}<<\Lambda_{m}\right) \\
& \sim V_{0} \log \frac{\left|\phi_{A D}\right|^{2}}{\Lambda_{m}^{2}}\left(\phi_{A D}>>\Lambda_{m}\right)
\end{aligned}
$$

where $\Lambda_{m}$ is the messenger scale. Of course the gravitational effect always exists, which lifts the flat direction by

$$
V_{G R A} \sim m_{3 / 2}^{2}\left|\phi_{A D}\right|^{2}
$$

Here the soft breaking mass $m_{\text {soft }}$ is much larger than the gravitino mass $m_{3 / 2}$. As the potential for the Affleck-Dine field is very flat at the large amplitude, the Q-ball formation is inevitable. In the gauge-mediated SUSY breaking models, the Q-ball formation makes the scenario difficult to produce sufficient baryon number of the universe while evading the cosmological problems. ${ }^{3}$

As we will discuss in the followings, the above-mentioned problems for the single-field models are naturally solved or modified in hybrid models. Here we briefly show the basics of the idea. In the conventional scenarios of Affleck-Dine baryogenesis, one should assume $H>m_{\phi_{A D}}$ before the time of baryogenesis so that the flat directions are destabilized by the gravitational corrections of $O(H)$, to obtain the large initial amplitude of the baryon-charged directions. This simple idea seems to solve the problem of the initial condition in the original model[2. On the other hand, however, this simple condition sometimes puts a severe constraint on the models, as we will discuss in the next section. To find a solution to the problem, we think it is interesting to invoke ideas that had been used to solve the problems in other cosmological scenarios. For example, when

\footnotetext{
${ }^{3}$ There are many discussions on this topic 8,9 .
} 
one considers chaotic inflation, problems arise for the original single-field model. The problems are solved by hybrid inflation, in which an additional field is included to lift the energy density and trigger the termination of inflation. Then it seems natural to ask, "Is it possible to find the trigger field in Affleck-Dine baryogenesis, which removes the serious constraints in the original single-field models?" In this paper we find the solution to the above (rather naive) question. In generic situations, it seems quite hard to find hybrid models in the conventional settings of supergravity. Thus we consider the scenario for the braneworld where the moduli for the brane distance can play the required role of the trigger field 11]. In this paper we focus our attention to the models with the low fundamental scale. The models with the low fundamental scale are attractive since the cosmological scenarios are expected to become quite different from the conventional one, which may leave distinguishable signatures in the present Universe. The Q-balls are naturally unstable in the models with large extra dimensions.

\section{Hybridized Affleck-Dine baryogenesis}

In this section we consider models for the braneworld in which the fundamental scale $\left(M_{*}\right)$ is much smaller than the conventional GUT scale. Naively, the model should be similar to the GMSB models, in which the gravitino mass is much smaller than the conventional soft mass, and the messenger scale is a cutoff scale for the effective theory. The crucial difference is the initial condensate of the Affleck-Dine field, which cannot become larger than the cutoff scale since the flat direction is localized on the brane. Of course in the conventional GMSB models, it becomes much larger than the messenger scale. As a result, flat potentials in GMSB models are destabilized until $H \sim m_{3 / 2}<<$ $m_{\text {soft }}$. On the other hand, in models with the low fundamental scale, the Affleck-Dine field starts to oscillate at $H \sim m_{\text {soft }}$ with the amplitude $<\phi_{A D}>\leq M_{*}$ unless there is the non-trivial mechanism to destabilize the potential. As is discussed in ref. [6], the situation is hopeless because the resultant baryon to entropy ratio is at most

$$
\begin{aligned}
\frac{n_{b}}{s} & \simeq \frac{n_{b}}{n_{\phi_{A D}}} \frac{T_{R}}{m_{\phi_{A D}}} \frac{\rho_{\phi_{A D}}}{\rho_{I}} \\
& <\frac{T_{R}}{m_{\phi_{A D}}}\left(\frac{m_{\phi_{A D}}^{2} M_{*}^{2}}{m_{\phi_{A D}}^{2} M_{p}^{2}}\right)
\end{aligned}
$$




$$
\sim 10^{-29}\left(\frac{M_{*}}{10^{6} \mathrm{GeV}}\right)^{2}
$$

for the reheating temperature $T_{R} \sim 1-10 \mathrm{MeV}$. Here $\rho_{I}$ denotes the energy density of the inflaton field. To avoid this difficulty, one should consider non-trivial realization of the Affleck-Dine mechanism. ${ }^{4}$

To avoid the above difficulty, we show the non-trivial mechanism to destabilize the flat direction on the brane. Here we consider the case in which the F-term on a brane destabilizes the Affleck-Dine flat direction. As in the models for brane inflation, we consider two branes separated at a distance. At the beginning of Affleck-Dine baryogenesis, these two branes are required to be located at a distance in the extra dimensions. We assume that in the true vacuum, the moduli for the brane distance is stabilized by the mass of $O\left(m_{3 / 2}\right)$, while it is destabilized by the $\mathrm{O}(\mathrm{H})$ gravitational corrections during inflation. Then the moduli is destabilized until $H \sim m_{3 / 2}$, which means that two branes are separated during this period. On one brane, for the simplest example, we assume the localized field $\mathrm{S}$ and the superpotential of the form[1]

$$
W_{1}=S \Lambda_{1}^{2}
$$

which spontaneously breaks supersymmetry by the F-term. On the other brane, a superfield $\Phi$ is localized with the superpotential $W_{2}=0$. When two branes are on top of each other, the localized fields $S$ and $\Phi$ may interact. Then finally in the true vacuum, where two branes are on top of each other, a superpotential appears on the brane,

$$
W_{1+2}=S\left(\Lambda_{1}^{2}-\Phi^{2}\right)
$$

which restores supersymmetry. One may expect many other forms of the superpotential[11]. The requirement for the mechanism is very simple. Supersymmetry is needed to be spontaneously broken when the interactions are absent, while it is restored in the vacuum. If the supersymmetry breaking terms induced by the above simple mechanism dominate the potential for the Affleck-Dine field, the Affleck-Dine field on

\footnotetext{
${ }^{4}$ In ref. [6], the Affleck-Dine field is put into the bulk where the large volume factor enhances the energy density of the Affleck-Dine field. In ref.[7], Affleck-Dine baryogenesis after thermal brane inflation [10] was considered. We have also discussed the effect of the cosmological defects in ref. [5].
} 
the brane is destabilized. In this case, the required trigger field is the moduli field that parametrizes the distance between branes.

In the above example, the oscillation of the Affleck-Dine field starts at $H \sim m_{3 / 2}<<$ $m_{\text {soft }}$. Since the oscillation starts much later than the conventional single-field models, the baryon to entropy ratio can be enhanced in our model. In the most optimistic case, when $\rho_{A D} \sim m_{\phi_{A D}}^{2}\left(\phi_{A D}^{i}\right)^{2}$,

$$
\frac{n_{B}}{s} \sim \frac{T_{R} m_{\phi_{A D}}^{2}\left(\phi_{A D}^{i}\right)^{2}}{m_{\phi_{A D}} \rho_{I}}
$$

where $T_{R}$ is the reheating temperature after Affleck-Dine baryogenesis, and $\phi_{A D}^{i}$ is the initial amplitude of $\phi_{A D}$. Then we obtain:

$$
\frac{n_{B}}{s} \sim 10^{-9}\left(\frac{T_{R}}{10 M e V}\right)\left(\frac{\left(10^{5} G e V\right)^{4}}{\rho_{I}}\right)\left(\frac{\phi_{A D}^{i}}{10^{6} G e V}\right)^{2}
$$

which is the most naive result, but is enough to explain the origin of the baryon asymmetry of the present Universe. The Hubble parameter when the AD oscillation starts is assumed to be $H_{o} \simeq m_{3 / 2}$. It is naturally assumed that the initial amplitude is as large as $\phi_{A D}^{i n i} \sim$ $M_{*}$. To be more precise, the baryon to entropy ratio is determined by the forms of the A-terms, which explicitly break baryon number ${ }^{5}$.

It is easy to see that our mechanism works for the models with the large fundamental scale (or small extra dimensions). In these models, however, there is no compelling motivation to consider hybrid models since (at this time) there seems no obvious advantage of the hybrid model. We think our model is stringent for the models with the low fundamental scale, where the conventional scenario utterly fails [5, 6, 7].

\footnotetext{
${ }^{5}$ See ref. 12] for more detail. Here we had not specified the phenomenological mechanism for supersymmetry breaking and its mediation, because we are not making a catalog in this paper. Although there are so many models in this direction, they are not directly related to the characteristic profiles of our model. Of course the produced baryon to entropy ratio depends on the A-terms. When the fundamental scale is much lower than the GUT scale, one should consider some non-trivial mechanisms to suppress the dangerous higher-dimensional terms that explicitly break the baryon number conservation. There are many discussions about the mechanism for suppressing the dangerous operators, which in turn determines the forms of the required A-terms. However, here we do not consult into the details of the arguments but simply assume that there is the required A-term when Affleck-Dine baryogenesis starts. Of course, if the required A-terms are completely forbidden by some gauged symmetries, it is impossible to produce any baryon number by using the Affleck-Dine mechanism. In ref. [5], we have already considered models in which the baryon number violating interactions are enhanced by the cosmological defects.
} 


\section{Inverted scenarios and other alternatives}

In this section we make some comments on the inverted scenarios and other alter-

natives of the above idea for Affleck-Dine baryogenesis. As in the models for hybrid inflaton[13, we can construct inverted scenarios 14] for our model. Perhaps the easiest way is;

1) Consider a source brane where a source field for the supersymmetry breaking is localized. In the true vacuum, this brane is located at a distance from the "SM-brane" where standard model fields are located.

2) The trigger field is the moduli for the distance between the source brane and the SM-brane. The potential for the moduli is destabilized in the true vacuum by the $O\left(m_{3 / 2}\right)$ correction, which makes these branes separated in the true vacuum.

3) During inflation and the follwing stage of the oscillation, the $O(H)>m_{3 / 2}$ correction stabilizes the moduli. Thus the branes stay on top of each other during this period.

4) When branes are located on top of each other, the corrections from the source brane destabilize some of the flat directions of the MSSM. During this period, the condensate of the Affleck-Dine field is expected.

5) As the Hubble parameter becomes small, the trigger field (the moduli for the brane distance) is destabilized. Then the branes move toward their minima. At this stage, the Affleck-Dine field is stabilized to start oscillation.

The model is quite similar to the original model that we have stated in the previous section, except for the mechanism for the supersymmetry breaking.

An alternative scenario for the inverted model is already discussed in ref. [7], in which Affleck-Dine baryogenesis after thermal brane inflation is discussed.

In ref.[11], a modified version of thermal brane inflation is discussed. As the thermal hybrid inflation starts at a distance, one can easily construct "not inverted" scenario for Affleck-Dine baryogenesis after thermal brane inflation. 


\section{Conclusions and Discussions}

At first, there had not been no concrete mechanism to arrange the suitable initial condition for the Affleck-Dine mechanism. Later observations [15] showed that the gravitational corrections of $O(H)$ can destabilize the flat direction to yield the expected initial condition for the mechanism. However, some later discussions suggested [3, 8] that this simple mechanism cannot work in its simplest form because of the non-trivial formation of the Q-balls or the thermal effects. Thus the succeeding scenarios are strongly model dependent. For models with large extra dimensions, the problem is quite serious for the original single-field models.

In this paper we extend the original single-field models to include an additional trigger field. The result is quite favorable for models with large extra dimensions. We have also constructed inverted scenarios and other alternatives.

\section{Acknowledgment}

We wish to thank K.Shima for encouragement, and our colleagues in Tokyo University for their kind hospitality.

\section{References}

[1] I.Antoniadis, N.A-Hamed, S.Dimopoulos, and G.R.Dvali, Phys.Lett.B436:257263,1998; I.Antoniadis, Phys.Lett.B246:377-384,1990; N.A-Hamed, S.Dimopoulos and G.R.Dvali, Phys.Lett.B429:263-272,1998.

[2] I.Affleck and M.Dine, Nucl.Phys.B249(1985)361

[3] A. Kusenko, Phys.Lett.B405:108,1997; A. Kusenko, M. E. Shaposhnikov, Phys.Lett.B418:46-54,1998. K. Enqvist, J. McDonald, Phys.Lett.B425:309-321,1998

[4] N. Arkani-Hamed, S. Dimopoulos, N. Kaloper, and J. March-Russell, Nucl.Phys.B567:189-228,2000; R. N. Mohapatra, A. Perez-Lorenzana, and C. A. de S. Pires, Phys.Rev.D62:105030,2000; A. M. Green and A. Mazumdar, 
Phys.Rev.D65:105022,2002; T. Matsuda, Phys.Rev.D66:107301,2002; D. H. Lyth, Phys.Lett.B448:191-194,1999; Phys.Lett.B466:85-94,1999.

[5] G.R. Dvali, G. Gabadadze, Phys.Lett.B460:47-57,1999; T. Matsuda, Phys.Rev.D66:023508,2002; Phys.Rev.D65:103502,2002; Phys.Rev.D65:107302,2002; Phys.Rev.D64:083512,2001; J.Phys.G27:L103-L108,2001; Phys.Lett. B486 (2000) 300305; A. Masiero, M. Peloso, L. Sorbo, and R. Tabbash, Phys.Rev.D62:063515,2000; A.Pilaftsis, Phys.Rev.D60:105023,1999; R.Allahverdi, K.Enqvist, A.Mazumdar and A.P-Lorenzana, Nucl.Phys. B618:377,2001; S. Davidson, M. Losada, and A. Riotto, Phys.Rev.Lett.84:4284-4287,2000.

[6] A.Mazumdar, Nucl.Phys.B597(2001)561, Phys.Rev.D64(2001)027304; A. Mazumdar and A. Perez-Lorenzana, Phys.Rev.D65:107301,2002; R. Allahverdi, K. Enqvist, A. Mazumdar, and A. Perez-Lorenzana, Nucl.Phys.B618:277-300,2001.

[7] T.Matsuda, Phys.Rev.D65:103501,2002;

[8] S. Kasuya, M. Kawasaki, Phys.Rev.D64:123515,2001; Phys.Rev.D62:023512,2000; K. Enqvist, A. Mazumdar, "Cosmological consequences of MSSM flat directions", hep-ph/0209244; M. Fujii, T. Yanagida, Phys.Rev.D66:123515,2002; M. Kawasaki, F. Takahashi, M. Yamaguchi, Phys.Rev.D66:043516,2002; M. Fujii, K. Hamaguchi, Phys.Rev.D66:083501,2002.

[9] A. Anisimov, M. Dine, Nucl.Phys.B619:729-740,2001; A. Anisimov, "Thermal Effects And Flat Direction Baryogenesis" hep-ph/0111233; S. Kasuya, M. Kawasaki, F. Takahash, "Affleck-Dine mechanism with negative thermal logarithmic potential" hep-ph/0302154.

[10] G.R. Dvali, Phys.Lett.B459:489-496,1999

[11] T.Matsuda, "Non-tachyonic brane inflation", hep-ph/0302035 " F-term, D-term and hybrid brane inflation", hep-ph/0302078; "Topological hybrid inflation in brane world" hep-ph/0302204; "Thermal hybrid inflation in brane world", hep-ph/0302253. Phys.Rev.D65:103501,2002; Phys.Lett. B423 (1998) 35-39;

[12] M.Dine, L.Randall, and S.Thomas, Nucl.Phys.B458(1996)291 
[13] A. D. Linde, Phys.Rev.D49:748-754,1994.

[14] E. J. Copeland, D. Lyth, A. Rajantie, M. Trodden, Phys.Rev.D64:043506,2001; D. H. Lyth, Ewan D. Stewart, Phys.Rev.D54:7186-7190,1996.

[15] M. Dine, W. Fischler and D. Nemechansky, Phys. Lett.B138 (1984) 169; G.D.Coughlan, R.Holman, P.Ramond and G.G.Ross, Phys. Lett. B140 (1984); S. Bertolami and G.G. Ross, Phys. Lett. B183 (1987) 163; E.D.Copeland, A.Liddle, D.Lyth, E.Stewart and D.Wands, Phys. Rev. D49 (1994) 6410; E.D.Stewart, Phys. Rev. D51 (1995) 6847; G. Dvali, Phys. Lett. B355 (1995) 78; M. Dine, L. Randall and S. Thomas, Phys. Rev. Lett. 75 (1995) 398; M. Dine, L. Randall and S. Thomas, Nucl. Phys. B458 (1996) 291. 